\title{
BIHARMONIC CURVES INTO QUADRICS
}

\author{
S. MONTALDO AND A. RATTO \\ Dipartimento di Matematica e Informatica, Università degli Studi di Cagliari \\ Via Ospedale 72 \\ 09124 Cagliari, Italia \\ e-mail:montaldo@unica.it; e-mail:rattoa@unica.it
}

(Received 20 June 2013; accepted 7 November 2013; first published online 26 August 2014)

\begin{abstract}
We develop an essentially algebraic method to study biharmonic curves into an implicit surface. Although our method is rather general, it is especially suitable to study curves in surfaces defined by a polynomial equation: In particular, we use it to give a complete classification of biharmonic curves in real quadrics of the threedimensional Euclidean space.
\end{abstract}

2000 Mathematics Subject Classification. 58E20

1. Introduction. Biharmonic curves $\gamma: I \subset \mathbb{R} \rightarrow(N, h)$ of a Riemannian manifold are the solutions of the fourth-order differential equation

$$
\nabla_{\gamma^{\prime}}^{3} \gamma^{\prime}-R\left(\gamma^{\prime}, \nabla_{\gamma^{\prime}} \gamma^{\prime}\right) \gamma^{\prime}=0
$$

where $\nabla$ is the Levi-Civita connection on $(N, h)$ and $R$ is its curvature operator.

As we shall detail in the next section, these arise from a variational problem and are a natural generalisation of geodesics. In the last decade, biharmonic curves have been extensively studied and classified in several spaces by analytical inspection of (1.1) (see, for example $[\mathbf{1 - 6 , 8}, \mathbf{9}, \mathbf{1 1}, \mathbf{1 5}]$ ).

Although much work has been done, the full understanding of biharmonic curves in a surface of the Euclidean three-dimensional space is far from being achieved. As yet, we have a clear picture of biharmonic curves in a surface only in the case that the surface is invariant by the action of one parameter group of isometries of ambient space. For example, in [3] it was proved that a biharmonic curve on a surface of revolution in the Euclidean space (invariant by the action of $S O(2)$ ) must be a parallel, that is, an orbit of the action of the group on the surface. This property was then generalised to invariant surfaces in a three-dimensional manifold [15].

The main obstacle in trying to describe and classify biharmonic curves in a surface by analytical methods is that (1.1) is the fourth-order differential equation, which is very hard to tackle.

In this paper, we propose a scheme to classify biharmonic curves into a quadric in the three-dimensional Euclidean space by using algebraic methods. The main point is that a quadric can be described implicitly by a polynomial equation $F(x, y, z)=$ 0 , and we will show that the biharmonic candidates must be the intersection of the given quadric with another specific algebraic surface $G(x, y, z)=0$. The latter property allows us to classify biharmonic curves into any non-degenerate quadric. 
In the last section we give some examples to show how to use this approach for other implicit surfaces.

2. Preliminaries. Harmonic maps are critical points of the energy functional

$$
E(\varphi)=\frac{1}{2} \int_{M}|d \varphi|^{2} d v_{g},
$$

where $\varphi:(M, g) \rightarrow(N, h)$ is a smooth map between two Riemannian manifolds $M$ and $N$. In analytical terms, the condition of harmonicity is equivalent to the fact that the map $\varphi$ is a solution of the Euler-Lagrange equation associated with the energy functional (2.1), i.e.

$$
\text { trace } \nabla d \varphi=0 \text {. }
$$

The left member of (2.2) is a vector field along the map $\varphi$, or, equivalently, a section of the pull-back bundle $\varphi^{-1}(T N)$ : it is called tension field and denoted as $\tau(\varphi)$.

A related topic of growing interest deals with the study of the so-called biharmonic maps: These maps, which provide a natural generalisation of harmonic maps, are the critical points of bienergy functional (as suggested by Eells and Lemaire in [7]),

$$
E_{2}(\varphi)=\frac{1}{2} \int_{M}|\tau(\varphi)|^{2} d v_{g} .
$$

Jiang in [12] derived the first variation and the second variation formulas for bienergy. In particular, he showed that the Euler-Lagrange equation associated with $E_{2}(\varphi)$ is

$$
\tau_{2}(\varphi)=-J(\tau(\varphi))=-\Delta \tau(\varphi)-\operatorname{traceR}^{\mathrm{N}}(\mathrm{d} \varphi, \tau(\varphi)) \mathrm{d} \varphi=0
$$

where $J$ denotes (formally) the Jacobi operator of $\varphi, \triangle$ is the rough Laplacian on the sections of $\varphi^{-1}(T N)$ that, for a local orthonormal frame $\left\{e_{i}\right\}_{i=1}^{m}$ on $M$, is defined by

$$
\Delta=-\sum_{i=1}^{m}\left\{\nabla_{e_{i}}^{\varphi} \nabla_{e_{i}}^{\varphi}-\nabla_{\nabla_{e_{i}}^{M} e_{i}}^{\varphi}\right\}
$$

and

$$
R^{N}(X, Y)=\nabla_{X} \nabla_{Y}-\nabla_{Y} \nabla_{X}-\nabla_{[X, Y]}
$$

is the curvature operator on $(N, h)$.

We point out that (2.4) is the fourth-order semi-linear elliptic system of differential equations. We also note that any harmonic map is an absolute minimum of bienergy, and so it is trivially biharmonic. Therefore, a general working plan is to study the existence of biharmonic maps which are not harmonic: these shall be referred to as proper biharmonic maps. We refer to [14] for existence results and general properties of biharmonic maps. 
Now, let $\gamma: I \rightarrow(N, h)$ be a curve parametrized by arc length from an open interval $I \subset \mathbb{R}$ to a Riemannian manifold. In this case, putting $T=\gamma^{\prime}$, the tension field becomes $\tau(\gamma)=\nabla_{T} T$ and the biharmonic equation (2.4) reduces to

$$
\nabla_{T}^{3} T-R\left(T, \nabla_{T} T\right) T=0 .
$$

In order to describe geometrically equation (2.6), let us recall the definition of the Frenet frame.

Definition 2.1 See, for example [13]. The Frenet frame $\left\{F_{i}\right\}_{i=1, \ldots, n}$ associated with a curve $\gamma: I \subset \mathbb{R} \rightarrow\left(N^{n}, h\right)$, parametrized by arc length, is the orthonormalisation of $(n+1)$-uple $\left\{\nabla_{\frac{\partial}{\partial t}}^{(k)} d \gamma\left(\frac{\partial}{\partial t}\right)\right\}_{k=0, \ldots, n}$ described by:

$$
\begin{aligned}
F_{1} & =d \gamma\left(\frac{\partial}{\partial t}\right), \\
\nabla_{\frac{\partial}{\partial t}}^{\gamma} F_{1} & =k_{1} F_{2}, \\
\nabla_{\frac{\partial}{\partial t}}^{\gamma} F_{i} & =-k_{i-1} F_{i-1}+k_{i} F_{i+1}, \quad \forall i=2, \ldots, n-1, \\
\nabla_{\frac{\partial}{\partial t}}^{\gamma} F_{n} & =-k_{n-1} F_{n-1},
\end{aligned}
$$

where the functions $\left\{k_{1}, k_{2}, \ldots, k_{n-1}\right\}$ are called the curvatures of $\gamma$, and $\nabla^{\gamma}$ is the Levi-Civita connection on the pull-back bundle $\gamma^{-1}(T N)$. Note that $F_{1}=T=\gamma^{\prime}$ is the unit tangent vector field along the curve.

Using the Frenet frame, the biharmonic equation (2.6) reduces to a differential system involving the curvatures of $\gamma$, and if we look for proper biharmonic solutions, that is for biharmonic curves with $k_{1} \neq 0$, we have

Proposition 2.2 ([2]). Let $\gamma: I \subset \mathbb{R} \rightarrow\left(N^{n}, h\right)(n \geq 2)$ be a curve parametrized by arc length from an open interval of $\mathbb{R}$ into an $n$-dimensional Riemannian manifold $\left(N^{n}, h\right)$. Then $\gamma$ is proper biharmonic if and only if:

$$
\left\{\begin{array}{l}
k_{1}=\text { constant } \neq 0 \\
k_{1}^{2}+k_{2}^{2}=R\left(F_{1}, F_{2}, F_{1}, F_{2}\right) \\
k_{2}^{\prime}=-R\left(F_{1}, F_{2}, F_{1}, F_{3}\right) \\
k_{2} k_{3}=-R\left(F_{1}, F_{2}, F_{1}, F_{4}\right) \\
R\left(F_{1}, F_{2}, F_{1}, F_{j}\right)=0 \quad j=5, \ldots, n
\end{array} .\right.
$$

As a special case of (2.7), if $\gamma: I \subset \mathbb{R} \rightarrow\left(N^{2}, h\right)$ is a curve into a surface, then $\gamma$ is proper biharmonic if and only if

$$
\left\{\begin{array}{l}
k_{1}=\text { constant } \neq 0 \\
k_{1}^{2}=K
\end{array},\right.
$$

where $K$ is the Gaussian curvature of surface $\left(N^{2}, h\right)$.

3. Formulas for the curvatures of implicit surfaces and implicit curves. Let $F$ : $\mathbb{R}^{3} \rightarrow \mathbb{R}$ be a differentiable function: We shall assume that, for all $p \in N^{2}=F^{-1}(0)$, $(\operatorname{grad} F)(p) \neq 0$ so that $N^{2}$ is a regular surface in $\mathbb{R}^{3}$. If we denote by $C_{H F}$ the cofactor 
matrix of the Hessian $H F$ of $F$, the Gaussian curvature of the surface $N^{2}$ is given by (see, for example [10])

$$
K=\frac{(\operatorname{grad} F)\left(C_{H F}\right)(\operatorname{grad} F)^{\top}}{\|\operatorname{grad} F\|^{4}} .
$$

Let now $F: \mathbb{R}^{3} \rightarrow \mathbb{R}$ and $G: \mathbb{R}^{3} \rightarrow \mathbb{R}$ be two differentiable functions such that $F^{-1}(0)$ and $G^{-1}(0)$ are, as above, two regular surfaces in $\mathbb{R}^{3}$, and also assume that at all points $p \in F^{-1}(0) \cap G^{-1}(0)$, the gradients $\operatorname{grad} F$ and $\operatorname{grad} G$ are linearly independent. Then $F^{-1}(0) \cap G^{-1}(0)$ defines the trace of a regular curve in $\mathbb{R}^{3}$ that locally can be parametrized by arc length as $\gamma(s)=(x(s), y(s), z(s)), s \in(a, b)$. The unit tangent vector to $\gamma$ is then

$$
\gamma^{\prime}(s)=\frac{d \gamma}{d s}=T=\frac{\operatorname{grad} F \wedge \operatorname{grad} G}{\|\operatorname{grad} F\|\|\operatorname{grad} G\|} .
$$

The curve $\gamma$ can be seen as a curve of both $F^{-1}(0)$ and $G^{-1}(0)$. For each point $p=\gamma(s), s \in(a, b)$, we denote by $k_{n}^{F}(p)$ (respectively $\left.k_{n}^{G}(p)\right)$ the normal curvature at $p$ of the surface $F^{-1}(0)$ (respectively $\left.G^{-1}(0)\right)$ in the direction of $T$.

The curvature $k(s)$ of the curve $\gamma:(a, b) \rightarrow \mathbb{R}^{3}$ can be computed in terms of the normal curvatures $k_{n}^{F}(p)$ and $k_{n}^{G}(p), p=\gamma(s)$, as

$$
k^{2}=\frac{1}{\sin ^{2} \vartheta}\left(\left(k_{n}^{F}\right)^{2}+\left(k_{n}^{G}\right)^{2}-2\left(k_{n}^{F}\right)\left(k_{n}^{G}\right) \cos \vartheta\right),
$$

where $\vartheta$ is the angle between $(\operatorname{grad} F)(p)$ and $(\operatorname{grad} G)(p)$, that is

$$
\cos \vartheta=\frac{\langle\operatorname{grad} F, \operatorname{grad} G\rangle}{\|\operatorname{grad} F\|\|\operatorname{grad} G\|} .
$$

The proof of (3.2) is immediate. In fact, $k(s)$ is the norm of $\gamma^{\prime \prime}(s)=d^{2} \gamma / d s^{2}$ which is normal to $T$. Thus,

$$
\gamma^{\prime \prime}=\alpha \frac{\operatorname{grad} F}{\|\operatorname{grad} F\|}+\beta \frac{\operatorname{grad} G}{\|\operatorname{grad} G\|}
$$

for some functions $\alpha, \beta:(a, b) \rightarrow \mathbb{R}$, which, recalling that

$$
k_{n}^{F}=\left\langle\gamma^{\prime \prime}, \frac{\operatorname{grad} F}{\|\operatorname{grad} F\|}\right\rangle, \quad k_{n}^{G}=\left\langle\gamma^{\prime \prime}, \frac{\operatorname{grad} G}{\|\operatorname{grad} G\|}\right\rangle,
$$

can be expressed by:

$$
\alpha=\frac{k_{n}^{F}-k_{n}^{G} \cos \vartheta}{\sin ^{2} \vartheta}, \quad \beta=\frac{k_{n}^{G}-k_{n}^{F} \cos \vartheta}{\sin ^{2} \vartheta} .
$$

Finally, looking at $\gamma(s)$ as a curve in the surface $F^{-1}(0)$, at a point $p=\gamma(s)$ the geodesic curvature $k_{1}(s)$, the normal curvature $k_{n}^{F}(p)$ and the curvature $k(s)$ are related by the formula

$$
k^{2}=k_{1}^{2}+\left(k_{n}^{F}\right)^{2} .
$$


Thus, combining (3.2) and (3.3), we have the following proposition.

Proposition 3.1. Let $F: \mathbb{R}^{3} \rightarrow \mathbb{R}$ and $G: \mathbb{R}^{3} \rightarrow \mathbb{R}$ be two differentiable functions such that $F^{-1}(0)$ and $G^{-1}(0)$ are two regular surfaces in $\mathbb{R}^{3}$. Assume that at all points $p \in F^{-1}(0) \cap G^{-1}(0)$ the gradients $\operatorname{grad} F$ and $\operatorname{grad} G$ are linearly independent. Then the geodesic curvature $k_{1}$ of the curve $\gamma:(a, b) \rightarrow F^{-1}(0) \subset \mathbb{R}^{3}$, with $\gamma(s) \in F^{-1}(0) \cap$ $G^{-1}(0)$, for all $s \in(a, b)$, is given by

$$
k_{1}^{2}=\frac{\left(\cos \vartheta k_{n}^{F}-k_{n}^{G}\right)^{2}}{\sin ^{2} \vartheta} .
$$

Moreover, taking twice the derivative of $F(\gamma(s))=0$, we find

$$
0=\frac{d^{2} F}{d s^{2}}=T(H F) T^{\top}+\left\langle\operatorname{grad} F, \gamma^{\prime \prime}\right\rangle
$$

It follows that

$$
k_{n}^{F}=-\frac{T(H F) T^{\top}}{\|\operatorname{grad} F\|}
$$

and, similarly,

$$
k_{n}^{G}=-\frac{T(H G) T^{\top}}{\|\operatorname{grad} G\|} .
$$

The main point here is that in order to compute geodesic curvature of curve $\gamma$ defined as in Proposition 3.1, there is no need to parametrize the intersection curve because (3.4) can be explicitly written in terms of $\operatorname{grad} F \operatorname{grad} G$ and the Hessian matrices $H F$ and $H G$.

4. Biharmonic curves into real quadrics. Let $\mathcal{Q}$ be a real, non-degenerate quadric in $\mathbb{R}^{3}$. Then, with respect to an adapted orthonormal frame of $\mathbb{R}^{3}, \mathcal{Q}=F^{-1}(0)$, where

$$
F(x, y, z)=\frac{x^{2}}{a^{2}}+\xi \frac{y^{2}}{b^{2}}+\zeta \frac{z^{2}}{c^{2}}-1, \quad \xi, \zeta= \pm 1 \text { and } a, b, c>0
$$

if $\mathcal{Q}$ is a quadric with centre, or

$$
F(x, y, z)=\frac{x^{2}}{a^{2}}+\eta \frac{y^{2}}{b^{2}}-2 z, \quad \eta= \pm 1 \text { and } a, b>0,
$$

otherwise.

According to (2.8), the Gauss curvature of the surface along a proper biharmonic curve must be a positive constant. If we compute the Gauss curvature of a quadric using (3.1), we get

$$
K=\frac{\xi \zeta}{a^{2} b^{2} c^{2}\left(\frac{x^{2}}{a^{4}}+\frac{y^{2}}{b^{4}}+\frac{z^{2}}{c^{4}}\right)^{2}}
$$


for the quadrics with centre and

$$
K=\frac{\eta}{a^{2} b^{2}\left(\frac{x^{2}}{a^{4}}+\frac{y^{2}}{b^{4}}+1\right)^{2}},
$$

otherwise. Thus, a quadric with centre can admit a proper biharmonic curve only if $\xi \zeta>0$. If $\xi=\zeta=1$ and $a=b=c$, then the quadric is a sphere, and the proper biharmonic curves are the circles of radius $\sqrt{2} a / 2$, a result proved in [3]. In all other cases, combining (2.8) and (4.3), we conclude that if there exists a proper biharmonic curve, then it must be the intersection of the given quadric with an ellipsoid of the type

$$
\frac{x^{2}}{a^{4}}+\frac{y^{2}}{b^{4}}+\frac{z^{2}}{c^{4}}=d^{2},
$$

where $d \in \mathbb{R}$. Similarly, a quadric without centre can admit a proper biharmonic curve only if $\eta>0$. In this case, the biharmonic curve, if there exists, must be the intersection of a given quadric with a cylinder of the type

$$
\frac{x^{2}}{a^{4}}+\frac{y^{2}}{b^{4}}=e^{2}-1,
$$

where $e \in \mathbb{R}$.

We are now in the right position to state the main result of the paper.

THEOREM 4.1. Let $\mathcal{Q}$ be a non-degenerate quadric which is not a sphere (if $\xi=\zeta=1$ in (4.1), without loss of generality we assume $a \geq b>c$ ).

(a) If $\mathcal{Q}$ is a quadric with centre (as in (4.1)), then $\mathcal{Q}$ admits a proper biharmonic curve if and only if

$$
\xi=\zeta=1 \text { and } a=b .
$$

Moreover, if (4.7) holds, the biharmonic curve is the intersection of quadric $\mathcal{Q}$ with the ellipsoid (4.5) with $d^{2}=1 /(a c)$.

(b) If $\mathcal{Q}$ is a quadric without centre (as in (4.2)), then $\mathcal{Q}$ does not admit any proper biharmonic curve.

Proof. We shall begin considering quadrics with centre. As we proved above, if there exists a proper biharmonic curve $\gamma$, it must be the intersection of $\mathcal{Q}$ with an ellipsoid (4.5), i.e.

$$
\gamma:\left\{\begin{array}{l}
F(x, y, z)=\frac{x^{2}}{a^{2}}+\xi \frac{y^{2}}{b^{2}}+\zeta \frac{z^{2}}{c^{2}}-1=0 \\
G(x, y, z)=\frac{x^{2}}{a^{4}}+\frac{y^{2}}{b^{4}}+\frac{z^{2}}{c^{4}}-d^{2}=0
\end{array},\right.
$$

with $\xi \zeta>0$. Suppose that $\xi=\zeta=1$ : then, using (3.4), we can compute the geodesic curvature of the intersection curve $\gamma$ as a curve of quadric $\mathcal{Q}$. A long, but 
straightforward, computation yields:

$$
k_{1}^{2}=\frac{1}{d^{2}} \frac{\left[d^{2} \lambda_{4}-\left(\frac{x^{2}}{a^{6}}+\frac{y^{2}}{b^{6}}+\frac{z^{2}}{c^{6}}\right) \lambda_{6}\right]^{2}\left[d^{2}\left(\frac{x^{2}}{a^{8}}+\frac{y^{2}}{b^{8}}+\frac{z^{2}}{c^{8}}\right)-\left(\frac{x^{2}}{a^{6}}+\frac{y^{2}}{b^{6}}+\frac{z^{2}}{c^{6}}\right)^{2}\right]}{,}
$$

where

$$
\lambda_{n}=a^{n} y^{2} z^{2}\left(b^{2}-c^{2}\right)^{2}+b^{n} x^{2} z^{2}\left(a^{2}-c^{2}\right)^{2}+c^{n} x^{2} y^{2}\left(a^{2}-b^{2}\right)^{2} .
$$

Now, since $\mathcal{Q}$ is not a sphere, we recall our hypothesis $a \geq b>c$ and also note that the curve $\gamma$ is a real curve with infinity points if and only if $d^{2} c^{2}-1>0$. Under these conditions the curve $\gamma$ can be parametrized by

$$
\gamma(u)=\left\{\begin{array}{l}
x(u)=r_{1} \cos u \\
y(u)=r_{2} \sin u \\
z(u)=c \sqrt{1-\left(r_{1}^{2} \cos ^{2} u\right) / a^{2}-\left(r_{2}^{2} \sin ^{2} u\right) / b^{2}}
\end{array}\right.
$$

where

$$
r_{1}=a^{2} \sqrt{\frac{1-c^{2} d^{2}}{a^{2}-c^{2}}}, \quad r_{2}=b^{2} \sqrt{\frac{1-c^{2} d^{2}}{b^{2}-c^{2}}}
$$

Now, replacing (4.10) in (4.9), we obtain

$$
k_{1}^{2}=\frac{8\left[A+4\left(c^{2} d^{2}-1\right) B \cos 2 u+\left(a^{2}-b^{2}\right)\left(c^{2} d^{2}-1\right) \cos 4 u\right]^{2}}{d^{2}\left(c^{2} d^{2}-1\right)\left[C+4 D \cos 2 u-\left(a^{2}-b^{2}\right)\left(c^{2} d^{2}-1\right) \cos 4 u\right]^{3}},
$$

where $A, B, C, D$ are real constants given by

$$
\begin{aligned}
A= & -8 a^{4} b^{4} d^{4}+8 a^{4} b^{2} d^{2}+3 a^{4} c^{2} d^{2}-3 a^{4}+8 a^{2} b^{4} d^{2}-6 a^{2} b^{2} c^{2} d^{2}-2 a^{2} b^{2} \\
& +3 b^{4} c^{2} d^{2}-3 b^{4}, \\
B= & a^{4}\left(2 b^{2} d^{2}-1\right)-2 a^{2} b^{4} d^{2}+b^{4}, \\
C= & -4 a^{4} b^{2} d^{2}+a^{4} c^{2} d^{2}+3 a^{4}-4 a^{2} b^{4} d^{2}-2 a^{2} b^{2} c^{2} d^{2}+2 a^{2} b^{2}+b^{4} c^{2} d^{2}+3 b^{4}, \\
D= & a^{4}\left(b^{2} d^{2}-1\right)-a^{2} b^{4} d^{2}+b^{4} .
\end{aligned}
$$

Next, by setting $w=\sin ^{2} u$ in (4.11), it is easy to conclude that, in terms of this new variable,

$$
k_{1}^{2}=\frac{N(w)}{D(w)}
$$

where the numerator and the denominator of (4.12) are polynomials of degrees 8 and 12 respectively. At this stage, direct inspection of the leading terms shows that $k_{1}$ can 
be a constant only if $a=b$. In this case, the condition that the curve $\gamma$ is proper biharmonic, that is $k_{1}^{2}-K=0$, becomes

$$
\frac{1-a^{2} c^{2} d^{4}}{a^{4} c^{2} d^{4}\left(c^{2} d^{2}-1\right)}=0
$$

from which the desired result follows.

When $\xi=\zeta=-1$, the computations are similar to the previous case. First, we point out that in this case $\gamma$ is a real curve with infinity points if and only if $a^{2} d^{2}-1>0$. Moreover, by means of a computation similar to (4.11), we can conclude that, if the geodesic curvature $k_{1}$ of $\gamma$ is constant, then $b=c$. Next, when $b=c$, the biharmonic condition $k_{1}^{2}-K=0$ now reads as

$$
\frac{a^{2} c^{2} d^{4}+1}{a^{2} c^{4} d^{4}\left(a^{2} d^{2}-1\right)}=0
$$

which has no real solution, so ending the case of quadrics with centre.

When $\mathcal{Q}$ is a quadric without centre, as in (4.2), a proper biharmonic curve $\gamma$, if it exists, must be, as we have remarked above, the intersection of $\mathcal{Q}$ with a cylinder, i.e.

$$
\gamma:\left\{\begin{array}{l}
F(x, y, z)=\frac{x^{2}}{a^{2}}+\frac{y^{2}}{b^{2}}-2 z=0, \\
G(x, y, z)=\frac{x^{2}}{a^{4}}+\frac{y^{2}}{b^{4}}-e^{2}+1=0,
\end{array}\right.
$$

where $e^{2}>1$. Now, our method leads us to the following expression for the geodesic curvature $k_{1}$ of $\gamma$ :

$$
k_{1}^{2}=\frac{\left(\lambda_{6}^{2}-a^{6} b^{6} e^{2} \lambda_{4}\right)^{2}}{e^{2}\left[\lambda_{8}+x^{2} y^{2}\left(a^{2}-b^{2}\right)^{2}\right]^{2}\left(a^{4} b^{4} e^{2} \lambda_{8}-\lambda_{6}^{2}\right)},
$$

where

$$
\lambda_{n}=b^{n} x^{2}+a^{n} y^{2} .
$$

In this case, we propose a purely algebraic inspection of (4.14) to show that $k_{1}^{2}$ is constant if and only if $a=b$. First we observe that the points

$$
P_{1}=\left(0, b^{2} \sqrt{e^{2}-1}, \frac{b^{2}}{2}\left(e^{2}-1\right)\right), \quad P_{2}=\left(a^{2} \sqrt{e^{2}-1}, 0, \frac{a^{2}}{2}\left(e^{2}-1\right)\right)
$$

belong to the connected curve $\gamma$. Next, direct substitution in (4.14) gives

$$
k_{1}^{2}\left(P_{1}\right)=\frac{\left(b^{2} e^{2}-a^{2}\left(e^{2}-1\right)\right)^{2}}{a^{8} e^{2}\left(e^{2}-1\right)}, \quad k_{1}^{2}\left(P_{2}\right)=\frac{\left(a^{2} e^{2}-b^{2}\left(e^{2}-1\right)\right)^{2}}{b^{8} e^{2}\left(e^{2}-1\right)} .
$$

Condition $k_{1}^{2}\left(P_{1}\right)=k_{1}^{2}\left(P_{2}\right)$ is equivalent to the second-degree equation in $e^{2}$ which admits no positive solution if $a \neq b$. Therefore, in this case $k_{1}^{2}$ cannot be a constant along $\gamma$. Conversely, if $a=b$, then $\lambda_{n}=a^{n}\left(x^{2}+y^{2}\right)=a^{n+4}\left(e^{2}-1\right)$ and $k_{1}^{2}$ is constant. 
Finally, under the hypothesis $a=b$, the biharmonicity condition, $k_{1}^{2}-K=0$, becomes

$$
\frac{1}{a^{4} e^{4}\left(e^{2}-1\right)}=0
$$

which has no solution.

5. Applications to other types of implicit surfaces. In this section, we discuss two examples where it is possible to apply the scheme used to classify biharmonic curves into quadrics.

EXAMPLE 5.1. Consider the implicit surface $N^{2}=F^{-1}(0)$, where

$$
F(x, y, z)=\frac{z^{2 n}}{c^{2}}+\left(x^{2}+y^{2}\right)^{n}-1, \quad c>0, n \geq 1 .
$$

Surface $N^{2}$ is a surface of revolution that for $n=1$ and $c \neq 1$ reduces to an ellipsoid. In this case, the curves with constant Gauss curvature are the parallels given by the intersection of surface $N^{2}$ with the planes $z=d=$ constant. Thus, unless $N^{2}$ is a sphere (i.e. $n=1=c$ ), the only possible biharmonic curves are

$$
\gamma:\left\{\begin{array}{l}
F(x, y, z)=\frac{z^{2 n}}{c^{2}}+\left(x^{2}+y^{2}\right)^{n}-1=0, \quad c>0, \\
G(x, y, z)=z-d=0, \quad d<\sqrt[n]{c} .
\end{array}\right.
$$

Using (3.1) we can compute the Gauss curvature of $N^{2}$ along $\gamma$ :

$$
K=\frac{c^{4}(2 n-1) A^{2 n} d^{2 n+2}\left(c^{2} A^{n}+d^{2 n}\right)}{\left(c^{4} d^{2} A^{2 n}+A d^{4 n}\right)^{2}}
$$

where

$$
A=\left(1-\frac{d^{2 n}}{c^{2}}\right)^{\frac{1}{n}}
$$

Next, computing the geodesic curvature of $\gamma$ by means of (3.4), we find

$$
k_{1}^{2}=\frac{c^{4} A^{2 n} d^{4 n}}{\left(c^{2}-d^{2 n}\right)^{2}\left(c^{4} d^{2} A^{2 n}+A d^{4 n}\right)} .
$$

Finally, the condition of biharmonicity, that is $k_{1}^{2}=K$, for a parallel (5.1) becomes:

$$
2 c^{4}(1-n) d^{2 n}+d^{6 n-2}\left(1-\frac{d^{2 n}}{c^{2}}\right)^{\frac{1-2 n}{n}}-c^{6}(2 n-1)\left(1-\frac{d^{2 n}}{c^{2}}\right)=0 .
$$

Although it is not easy to write down the explicit solutions of (5.2) as a function $d=d(n, c)$, we point out that (5.2) admits a solution $d_{0} \in[0, \sqrt[n]{c})$ for any $c>0$ and $n \geq 1$. To see this, we observe that the left-hand side of (5.2) is continuous in $d$, assumes a negative value for $d=0$ and diverges to $+\infty$ as $d$ tends to $\sqrt[n]{c}$. 
EXAMPLE 5.2. In this example we consider the case of graphs of revolution. Thus, we assume that $N^{2}=F^{-1}(0)$ with

$$
F(x, y, z)=z-f\left(\sqrt{x^{2}+y^{2}}\right),
$$

for some differentiable function $f$. As in the previous example, the only curves such that the restriction of the Gauss curvature of $N^{2}$ is constant are the parallels $z=$ $d=$ constant. If we put $\rho=\sqrt{x^{2}+y^{2}}$, the Gauss curvature of $N^{2}$ along a parallel $z=d=f(\rho)$ and the geodesic curvature are respectively

$$
K=\frac{f^{\prime}(\rho) f^{\prime \prime}(\rho)}{\rho\left(f^{\prime}(\rho)^{2}+1\right)^{2}}, \quad k_{1}^{2}=\frac{1}{\rho^{2}\left(f^{\prime}(\rho)^{2}+1\right)} .
$$

It follows that a parallel $\rho=\rho_{0}$ is biharmonic if and only if

$$
f^{\prime}\left(\rho_{0}\right)^{2}-\rho_{0} f^{\prime}\left(\rho_{0}\right) f^{\prime \prime}\left(\rho_{0}\right)+1=0 .
$$

Moreover, if $f$ is a solution of the ordinary differential equation (ODE)

$$
f^{\prime}(\rho)^{2}-\rho f^{\prime}(\rho) f^{\prime \prime}(\rho)+1=0,
$$

then all the parallels are biharmonic. The solution of (5.4) can be explicitly computed, and is given by

$$
f(\rho)=\frac{1}{2}\left(\rho \sqrt{e^{2 c_{1}} \rho^{2}-1}-e^{-c_{1}} \log \left(2 e^{c_{1}}\left(\sqrt{e^{2 c_{1}} \rho^{2}-1}+e^{c_{1}} \rho\right)\right)\right)+c_{2}, \quad c_{1}, c_{2} \in \mathbb{R} .
$$

We remark that the surface of revolution with the property that all its parallels are biharmonic was already found in [3] using different methods, and afterwords Monterde in $[\mathbf{1 6}]$ proved that it is the only surface in $\mathbb{R}^{3}$ with the property that all the level curves of the Gauss curvature are proper biharmonic and the gradient lines of the Gauss curvature are geodesics.

ACKNOwLEDGEMENT. This work was supported by P.R.I.N. 2010/11 - Varietà reali e complesse: geometria, topologia e analisi armonica and INdAM - Italy.

\section{REFERENCES}

1. R. Caddeo, S. Montaldo and C. Oniciuc, Biharmonic submanifolds of $\mathbb{S}^{3}$, Int. J. Math. 12 (2001), 867-876.

2. R. Caddeo, S. Montaldo, C. Oniciuc and P. Piu, The Euler-Lagrange method for biharmonic curves, Mediterr. J. Math. 3 (2006), 449-465.

3. R. Caddeo, S. Montaldo and P. Piu, Biharmonic curves on a surface, Rend. Mat. Appl. 21 (2001), 143-157.

4. R. Caddeo, C. Oniciuc and P. Piu, Explicit formulas for non-geodesic biharmonic curves of the Heisenberg group, Rend. Sem. Mat. Univ. Politec. Torino 62 (2004), 265-277.

5. J. T. Cho, J-I. Inoguchi and J-E. Lee, Biharmonic curves in 3-dimensional Sasakian space forms, Ann. Mat. Pura Appl. 186 (2007), 685-701.

6. I. Dimitric, Submanifolds of $\mathbb{E}^{m}$ with harmonic mean curvature vector, Bull. Inst. Math. Acad. Sinica 20 (1992), 53-65. 
7. J. Eells and L. Lemaire, Selected topics in harmonic maps, CBMS Regional Conference Series in Mathematics, vol. 50 (American Mathematical Society, Providence, RI 1983).

8. D. Fetcu, Biharmonic-Legendre curves in Sasakian space forms, J. Korean Math. Soc. 45 (2008), 393-404.

9. D. Fetcu, A note on biharmonic curves in Sasakian space forms, Ann. Mat. Pura Appl. 189 (2010), 591-603.

10. R. Goldman, Curvature formulas for implicit curves and surfaces, Comput. Aided Geom. Des. 22 (2005), 632-658.

11. J-I. Inoguchi and J-E. Lee, Affine biharmonic curves in 3-dimensional homogeneous geometries, Mediterr. J. Math. 10 (2013), 571-592

12. G. Y. Jiang, 2-harmonic maps and their first and second variation formulas, Chin. Ann. Math. A 7 (1986), 389-402. 1965).

13. D. Laugwitz, Differential and Riemannian geometry (Academic Press, Waltham, MA,

14. S. Montaldo and C. Oniciuc, A short survey on biharmonic maps between Riemannian manifolds, Rev. Un. Mat. Argentina 47 (2006), 1-22.

15. S. Montaldo and I. I. Onnis, Biharmonic curves on an invariant surface, J. Geom. Phys. 59 (2009), 391-399.

16. J. Monterde, Surfaces with a family of non-geodesic biharmonic curves, Rend. Mat. Appl. 28 (2008), 123-131. 